\title{
A Passive Imaging System for Geometry Measurement for the Plasma Arc Welding Process
}

\author{
Tomas Font comas, Chenglei Diao, Jialuo Ding, Stewart Williams, and Yifan Zhao
}

\begin{abstract}
Automatic and flexible geometry measurement of the weld pool surface can help better understand the complex welding processes and even provide feedback to better control this process. Most of existing imaging systems use an additional source of illumination to remove the light interference coming from the welding arc but it is usually costly. This paper introduces a novel low-cost optical-sensor-based monitoring system working under passive mode to monitor the wire + arc additive manufacture process, particularly for plasma arc welding. Initially, configurations and parameters of camera are investigated to achieve good visualization of weld pool. A novel camera calibration methodology using the nozzle of a computer numerical control (CNC) machine is then proposed for this imaging system, allowing estimation of the camera position with respect to the inspecting surface and its orientation in an easy-to-use approach. The verification tests show that the average error of the calibration is less than 1 pixel. As a case study, an image analysis routine is proposed to measure the width of the bead during the welding process. The results show that the proposed system is effective to measure the dimension of weld pool.
\end{abstract}

Index Terms-Additive manufacturing (AM), camera calibration, edge detection, plasma arc welding (PAW), wire + arc additive manufacture (WAAM).

\section{INTRODUCTION}

A DDITIVE manufacturing (AM) is a process of threedimensional (3-D) printing in which parts are built by adding materials layer by layer. Compared to traditional subtrac-

Manuscript received November 8, 2016; revised January 24, 2017 and February 14, 2017; accepted March 7, 2017. Date of publication March 22, 2017; date of current version August 9, 2017. This work was supported in part by the Through-Life Engineering Service Centre, in part by the Welding Engineering and Laser Processing Centre, and in part by the Computational and Software Techniques in Engineering M.Sc. Course at Cranfield University, Cranfield, U.K. (Corresponding author: Yifan Zhao.)

T. Font comas is with the Computational and Software Techniques in Engineering M.Sc. Course, Cranfield University, Cranfield, MK43 OAL, U.K. (e-mail: tfontcomes@gmail.com).

C. Diao, J. Ding, and S. Williams are with the Welding Engineering and Laser Processing Centre, Cranfield University, Cranfield, MK43 OAL, U.K. (e-mail: C.Diao@ cranfield.ac.uk; jialuo.ding@ cranfield.ac.uk; s.williams@cranfield.ac.uk).

Y. Zhao is with the Through-Life Engineering Service Centre, Cranfield University, Cranfield, MK43 0AL, U.K. (e-mail: yifan.zhao@ cranfield.ac.uk).

Color versions of one or more of the figures in this paper are available online at http://ieeexplore.ieee.org.

Digital Object Identifier 10.1109/TIE.2017.2686349 tive manufacturing technologies, this technique allows a high reduction in costs by substantially saving material and reducing lead time. Additionally, it offers higher flexibility in the design. One of the most promising techniques of AM under development is wire + arc additive manufacture (WAAM), which uses an electric arc as the power source and wire as the feedstock. WAAM is well suited of manufacturing medium- to large-scale parts and is gaining great interest in sectors such as the aerospace industry, especially where the need of manufacturing titanium parts is growing significantly because of its chemical compatibility with composites. Additionally, it offers a cleaner working environment, higher deposition rates, and less risk of contamination in comparison with powder-based systems [1].

Fluctuations of parameters and working conditions in the WAAM process can have a great influence on the dimensions and geometry of the final piece. The main factors requiring significant investigation include changes in operating parameters, heat dissipation, interlayer temperature, and quality of the previous layer. To deal with these, the process should be monitored and ideally controlled. A promising approach is to use vision techniques to track the weld pool geometry allowing monitoring of the process or potentially implementing a closed-loop control system. Few research works have been reported in the field of AM regarding optical sensing and corresponding reliable measurement, but several techniques have been implemented for the automation and enhancement of welding processes.

Obtaining high-quality images of the weld pool during the WAAM process is challenging due to the harsh conditions during the melting and deposition of the metal. The high temperature of the arc causes high intensity light radiation, which interferes with the image acquisition process. Kovacevic et al. [2] proposed to use a high-shutter-speed camera synchronized with a pulsed laser as an additional source of illumination in gas tungsten arc welding. The system works because the laser is monochromatic so the camera has a filter that is opaque to all radiation except at the laser wavelength. This greatly reduces the intensity of the plasma light so that the laser light can be observed. Because of the high cost of the illumination equipment, including the camera head, laser unit, and controller, a solution to use an array of low-cost semiconductor laser diodes and an appropriate bandpass filter matching the laser wavelength has been proposed by Vilarinho et al. [3]. Recently, a few ways of reconstructing the 3-D weld pool geometry assisted by a laser have been developed [4]-[6]. In these methods, an addi- 
tional source of light is used for measuring the weld pool shape indirectly, rather than reducing the arc interference. Using a laser with a dot matrix structured light pattern, the weld pool surface can be used as a specular mirror to reflect the light into an image plane. The camera records the plane where the laser dots are reflected and not the weld pool directly. An imaging system for WAAM working under the passive mode attracts less interests than the active mode. It uses the arc light to illuminate the scene; however, this process requires an accurate calibration of the camera position and a precise selection of a narrow-band filter to reduce the arc light [7]. A few research works using passive vision have been reported to enhance and automate welding processes [8], [9], or extract different features [10]-[12] from the weld pool for gas metal arc welding (GMAW) based AM. Recent research from Zhan et al. [13] introduced a method of using passive vision system for monitoring the wire deviation during the WAAM process. Additionally, infrared (IR) cameras have been used to monitor the weld pool [14], [15]; however, interference coming from the arc causes a big problem to obtain high-quality IR images.

In terms of the properties to measure using a camera, most of these research works focus on the step height between subsequence layers [16], the width of bead [11]-[17], and the height (nozzle to top surface distance) [11]-[17]. Accurate measurement of these properties is critical to have a better control of the WAAM process based on the input parameters, such as the arc current, the wire feeding rate, and the travel speed, etc. Although identification of the optimal properties to monitor the weld pool is not the scope of this paper, a case study using the proposed imaging system to measure the width of the weld pool is presented.

Plasma arc welding (PAW) has fewer transfer modes than GMAW processes due to the metal wire not being the electrode and hence it is a more stable process. It also allows a higher deposition rate and greater effective wall width compared to other additive layer manufacturing processes [18]. Due to these advantages, it is believed that PAW has great potential and will attract more and more interests in future. PAW has a very bright arc compared to GMAW; this will greatly increase the complexity in image processing. To face this challenge, developing a reliable and tailored weld pool monitoring system is therefore timely and highly demanded. To our knowledge, very few passive imaging systems have been developed for the PAW process due to the challenge of lighting condition and calibration. This paper introduces a flexible optical-sensor-based monitoring system without additional lighting sources to measure the geometry of weld pool in the PAW process. To accommodate the measurement of different properties, an easy-to-use calibration routine is developed to convert the measurement on image plane to the world coordinate system. Such research will be particularly useful for imaging systems based on a single camera that is required to change position regularly to measure different properties or based on multiples cameras at different positions.

\section{IMAGING SYSTEM SETUP}

In order to implement the PAW process, a CNC machine, a plasma welding power source, and a wire feeder are combined.

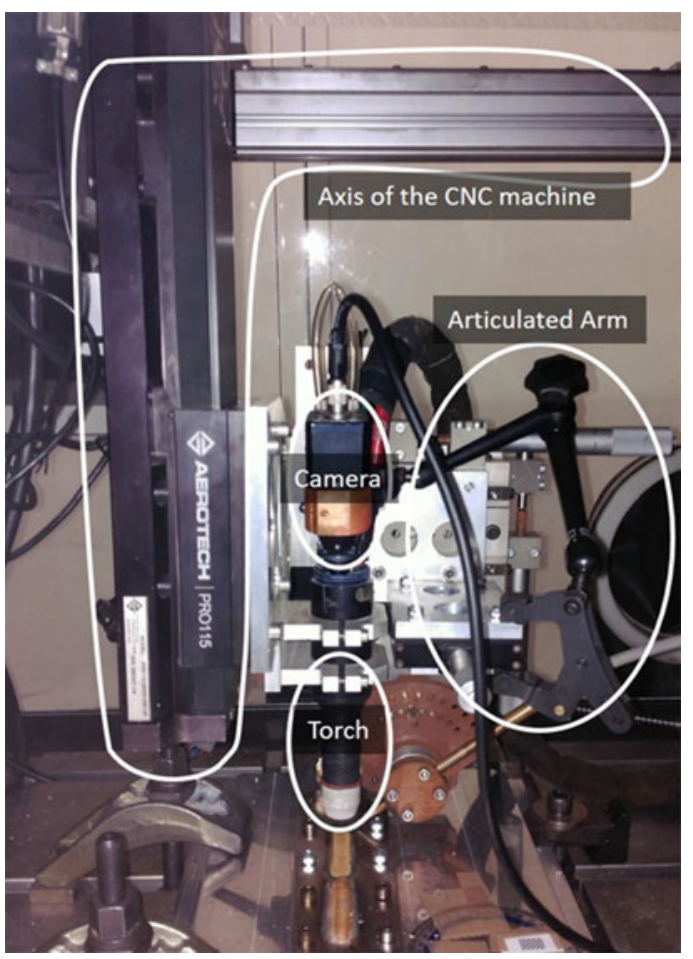

Fig. 1. Experimental layout.

Additionally, the MC500S Weld Vision Camera System, a special welding camera, is used for monitoring the process. The camera is connected to a video controller module, which allows changing gain, intensity, and shutter speed. As illustrated in Fig. 1, the camera is mounted on the moving panel of this CNC gantry, held by an articulated arm. The torch is mounted on the same moving panel of the CNC machine so that relative position between the camera and the torch remain the same during the deposition process. All the equipment mentioned above are setup inside a chamber. The chamber is used to create an inert atmosphere, with argon gas, to avoid the oxidation of titanium while the deposition is being processed. Due to the fact that the torch is very close (about $8 \mathrm{~mm}$ ) to the substrate during welding process, which the camera will be calibrated toward, LEDs used at the corners light will most likely be blocked by the torch and the CNC machine. Such setting will introduce shadows in the calibration grid, which will make the image processing more complicated. It will also introduce additional cables into the already crowded CNC machine.

For measuring a specific property from images, one important aspect that determines the quality of images is the location of the camera. To find out the best configuration of the camera, several experiments were conducted with different camera positions, as illustrated in Fig. 2. With the left setup, the wire is visible, but the visual quality of the weld pool is poor due to the strong arc light and the specular surface of the weld pool. In the middle image, the wire can be clearly observed but the visualization of the weld pool is still greatly affected by arc light. The right setup provides the best view of the weld pool with less reflection, but the wire is not visible. The case study of this paper focuses on the measurement of the width of the weld pool only, so the right setup was used. 

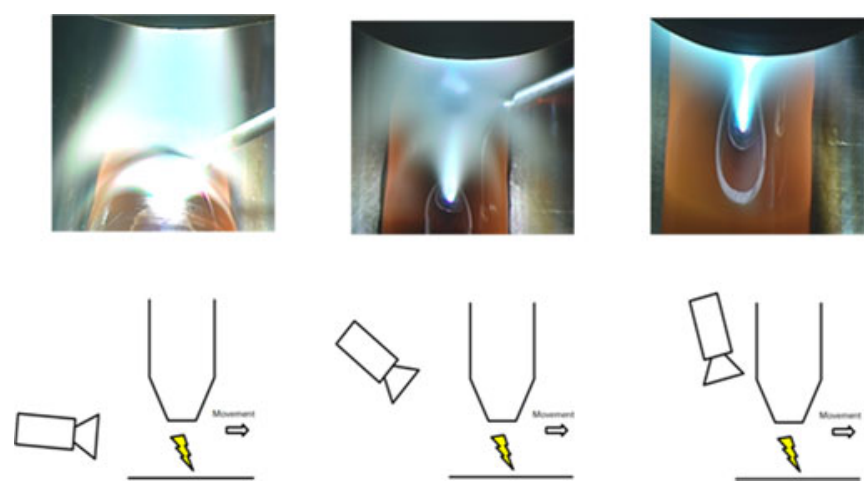

Fig. 2. Captured images with respect to different camera positions.

\section{Proposed Calibration and Measurement System}

\section{A. Mathematical Background of Camera Calibration}

The camera calibration is the process of estimating the intrinsic and distortion parameters of the imaging sensor and lens, and extrinsic parameters with respect to the object for measurement. These parameters define how a point in the 3-D world is projected into the image plane, and they are the key to measure the geometry of an object, correct lens distortions, or find the position of the camera. Without considering distortion, the equation

$$
\left[\begin{array}{c}
u_{i} \\
v_{i} \\
1
\end{array}\right]=K \cdot\left[\begin{array}{ll}
R_{\mathrm{CW}} & t_{\mathrm{CW}}
\end{array}\right] \cdot\left[\begin{array}{c}
X_{c} \\
Y_{c} \\
Z_{c} \\
1
\end{array}\right]
$$

maps any 3-D point in the world coordinate system to the image plane, where $\left\{u_{i}, v_{i}\right\}$ are the ideal coordinates in the unit of pixel of the image and $\left\{X_{c}, Y_{c}, Z_{c}\right\}$ is a 3-D point in the world coordinate system. The symbol $K$ corresponds to a $3 \times 3$ matrix containing the intrinsic parameters, and $\left[\begin{array}{ll}R_{\mathrm{CW}} & t_{\mathrm{CW}}\end{array}\right]$ corresponds to a $3 \times 4$ matrix containing the extrinsic parameters (a rotation matrix and a translation vector), which denotes the transformation from the 3-D world coordinate to the 3-D camera coordinate. Equivalently, the extrinsic parameters define the position of the camera center and the camera's heading in the world coordinate.

In photography, distortion is generally referred to as an optical aberration that deforms and bends physically straight line and makes them curvy in images. The distortion is caused by the lens. Normally distortion can be described by five parameters

$$
\left\{\begin{array}{lllll}
k_{1} & k_{2} & k_{3} & p_{1} & p_{2}
\end{array}\right\}
$$

where $k_{1}, k_{2}$, and $k_{3}$ are radial parameters, and $p_{1}$ and $p_{2}$ are tangential parameters. The models to correct distortion can be written as

$u_{r}=\left(1+k_{1} r^{2}+k_{2} r^{4}+k_{3} r^{6}\right) u_{i}+2 p_{1} u_{i} v_{i}+p_{2}\left(r^{2}+2 u_{i}^{2}\right)$,

$v_{r}=\left(1+k_{1} r^{2}+k_{2} r^{4}+k_{3} r^{6}\right) v_{i}+2 p_{2} u_{i} v_{i}+p_{1}\left(r^{2}+2 v_{i}^{2}\right)$

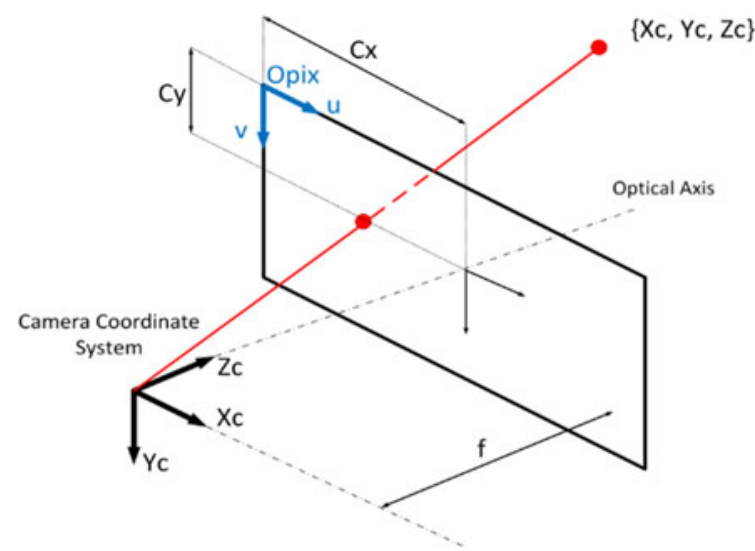

Fig. 3. Pinhole camera model with intrinsic parameters.

where $\left\{u_{r}, v_{r}\right\}$ are the true coordinate in images and $r$ is the radial distance of an ideal pixel to the center of the image, expressed as

$$
r^{2}=u_{i}^{2}+v_{i}^{2}
$$

Usually, only radial distortion is considered and tangential parameters are set to zero.

Intrinsic parameters remain constant once the camera and lens is integrated and focused. If the focal length is changed, intrinsic parameters will also change. Intrinsic parameters model the pinhole camera model, which projects any 3-D point from a coordinate system fixed with respect to the camera into the image plane. The matrix of the intrinsic parameters can be written as

$$
K=\left[\begin{array}{ccc}
f_{x} & 0 & C_{x} \\
0 & f_{y} & C_{y} \\
0 & 0 & 1
\end{array}\right]
$$

where $f_{x}$ and $f_{y}$ denote the focal length in the $x$ and $y$ directions, respectively. They are in pixel unit and usually can be simplified to $f_{x}=f_{y}=f$, assuming square pixels. $C_{x}$ and $C_{y}$ correspond to an offset to move the centerof the image to the upper left corner. Fig. 3 illustrates these parameters graphically.

The extrinsic parameters consist of a homogeneous transformation, which include a rotation and a translation operation. The translation operation consists of vectors from three dimensions, described as

$$
t=\left\{\begin{array}{lll}
t_{x} & t_{y} & t_{z}
\end{array}\right\}
$$

A rotation operation has 3-degrees of freedom corresponding to three angles of rotation, one for each of the axis. In this paper, the Euler angles [19] are employed to describe a rotation operation, which can be written as

$$
R=R_{z}(\phi) R_{y}(\theta) R_{x}(\psi)
$$

where $R_{z}(\phi)$ corresponds to a rotation of $\phi$ degree around the $z$-axis, $R_{y}(\theta)$ corresponds to a rotation of $\theta$ degree around the $y$-axis, and $R_{x}(\psi)$ corresponds to a rotation of $\psi$ degree around 
the $x$-axis. Then, (8) can be rewritten as

$$
\begin{aligned}
& R= \\
& {\left[\begin{array}{ccc}
\cos \theta \cos \phi & \sin \psi \sin \theta \cos \phi-\cos \psi \sin \phi & \cos \psi \sin \theta \cos \phi+\sin \psi \sin \phi \\
\cos \theta \sin \phi & \sin \psi \sin \theta \sin \phi+\cos \psi \cos \phi & \cos \psi \sin \theta \sin \phi-\sin \psi \cos \phi \\
\sin \theta & \sin \psi \cos \theta & \cos \psi \cos \theta
\end{array}\right] .}
\end{aligned}
$$

Note that the extrinsic parameters depend upon the location of the camera. Any move of the camera will require a recalculation of these parameters.

\section{B. Calibration Methodology}

The proposed calibration method requires taking several pictures of a calibration pattern from different points of view to improve the accuracy. This paper uses a checkerboard, which is a well-accepted calibration pattern in many research [20], [21]. If the size of the checkerboard square is known, the 3-D world coordinate of the points on the grid can be also inferred by putting the world coordinate system on it. The calibration process consists of solving a system of equations to find the intrinsic, distortion, and extrinsic parameters, which are the ones that relate any 3-D world point with a pixel position on the image plane. Hence, by knowing the 3-D world coordinate of the checkerboard points and knowing the 2-D pixel position corresponding to each of these points, a system of equations can be solved to find all the necessary parameters. Note that theoretically only one pair of 3-D-2-D points would be needed for each parameter, but as aforementioned increasing the number of points the error can be reduced by averaging the results.

Usually, when calibrating a camera, the procedure is simply moving a calibration pattern in front of a fixed camera. In this application, a few problems arose when trying this approach.

1) The camera has a narrow field of view so the calibration pattern must be very small $(15 \mathrm{~mm} \times 10.5 \mathrm{~mm})$. Small hand shakings can make it difficult to move the pattern.

2) Once the camera is fixed, there are many elements such as the $\mathrm{CNC}$ machine, the torch, or the chamber itself that would limit the movement of the calibration grid.

3) The fact that all equipment is inside a chamber leads to a lack of light. If the grid is moved, a lamp to illuminate it must be moved too, which will make this process very complicated.

To overcome these problems, instead of moving the calibration pattern with respect to the camera, in this paper, the camera will be moved with respect to the calibration pattern, which will remain fixed. Although such routine has been used in calibration of many applications, its application in PAW monitoring is new. Furthermore, to estimate the camera's position, the proposed methodology uses the position of the nozzle with respect to the world origin, which can be obtained by using the position of the nozzle displayed by the CNC machine interface. It is noted that the problems listed in the above three bullet points sometimes do not apply, because internal camera calibration usually does not need to be conducted online. It is an offline step that does

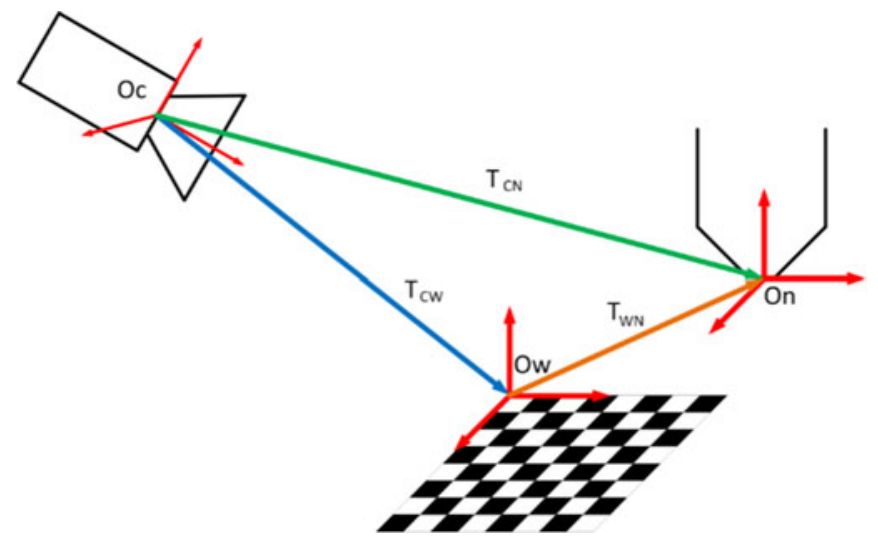

Fig. 4. Representation of the coordinate systems and transformations involved in measuring the camera pose.

not depend on the surrounding equipment (except for adjusting the lens).

The proposed calibration routine can be summarized as follows.

1) Place the calibration grid on the plane where the deposition process will be undertaken.

2) Calculate the position of the world origin in the $\mathrm{CNC}$ coordinate.

3) Focus the camera considering its location.

4) Move the camera to capture pictures of the calibration grid from different angles of view. While moving the camera, the difference between two consecutive pictures should be noticeable. It is suggested to take pictures when the row lines and column lines of the grid are not parallel in the image. A picture from exactly the top of the grid, where the lines are parallel, will contain less information.

5) Estimate the intrinsic and distortion coefficients using the captured pictures in step 4.

6) Fix the camera on the position where the recording of the process will be conducted, and take a picture of the calibration pattern and record the position of the $\mathrm{CNC}$ machine.

7) Estimate the extrinsic parameters using the captured picture in step 6.

\section{Camera Localization}

The quality of obtained images usually depends on the location of the camera. The proposed imaging system also allows an automatic localization of the camera. The position of the camera with respect to the nozzle will not change, because the camera is held with an articulated arm mounted onto the moving system. Because the calibration grid can be fixed wherever is convenient, the position of the camera must be calculated with respect to a point on the moving elements of the $\mathrm{CNC}$ machine. The solution of this system is to use an intermediate coordinate system on the nozzle. It can be seen from Fig. 4 that the blue transformation is composed by the extrinsic parameters obtained in step 7 of the calibration routine. It is a rotation and a translation transformation to transform the camera coordinate 
system into the world coordinate system. The orange transformation transforms the world coordinate system into the nozzle coordinate system. It can be estimated by calculating the difference between the position of the CNC machine in the world origin and the recorded position of the CNC machine in step 6 . Note that this transformation will consist only of a translation between the two coordinates systems, but no rotation.

Knowing the blue transformation matrix

$$
T_{\mathrm{CW}}=\left\{\begin{array}{ll}
R_{\mathrm{CW}} & t_{\mathrm{CW}}
\end{array}\right\}
$$

and the orange transformation matrix

$$
T_{\mathrm{WN}}=\left\{\begin{array}{ll}
R_{\mathrm{WN}} & t_{\mathrm{WN}}
\end{array}\right\}
$$

the green transformation matrix

$$
T_{\mathrm{CN}}=\left\{\begin{array}{ll}
R_{\mathrm{CN}} & t_{\mathrm{CN}}
\end{array}\right\}
$$

which corresponds to the location of the nozzle with respect to the camera, can be estimated by

$$
T_{\mathrm{CN}}=T_{\mathrm{CW}} \cdot T_{\mathrm{WN}} .
$$

The homogeneous transformation is a $4 \times 4$ matrix composed by a $3 \times 3$ rotation matrix, a $3 \times 1$ translation vector, and the scaling factor.

Once the nozzle position in the camera coordinate (green transformation) is estimated, the last step is to invert this transformation to obtain the position of the camera with respect to the nozzle coordinate system. Note that the camera coordinate system is always defined with the z-axis pointing to the direction in which the camera is recording. To reduce computational time, without inverting the $4 \times 4$ transformation matrix, the camera rotation matrix and translation vector can be calculated based on the green transformation described by (14) and (15), respectively,

$$
\begin{aligned}
R_{\mathrm{NC}} & =\left(R_{\mathrm{CN}}\right)^{T} \\
t_{\mathrm{NC}} & =-R_{\mathrm{NC}} \cdot t_{\mathrm{CN}} .
\end{aligned}
$$

\section{Measuring Real Distance}

The main problem encountered during traducing the measure from pixels to real world units is that when taking a picture and projecting the 3-D coordinates of an object into the image plane (2-D), there is a loss of information, particularly the depth. For every pixel in the image there is an infinite amount of possible locations in the 3-D space. To measure distances and angles on the images, recovering this lost information is essential.

Stereo vision is a well-known technique to address this problem. It uses the principle of having the same two points in both images, and therefore finding the intersection of the two pixel rays the 3-D depth information can be recovered. In the present case, this technique is not possible because only a single camera is used. Hence, some extra geometrical information is needed to find the 3-D coordinate from a 2-D pixel. The extra information that can be used is the plane equation in which we know the desired pixel will be contained. For instance, in case of measuring

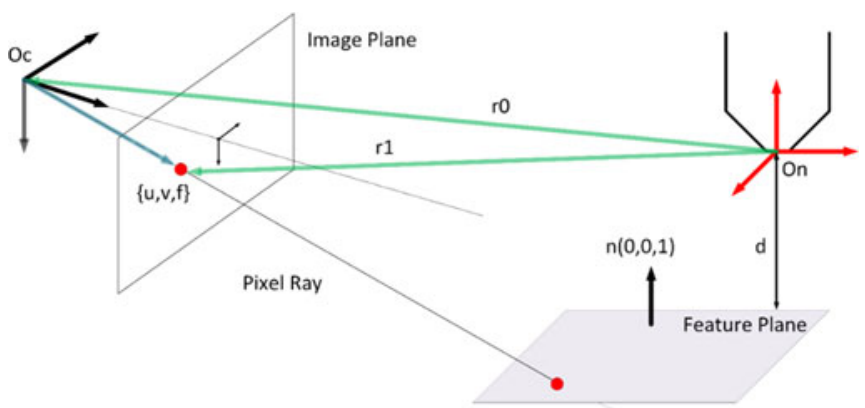

Fig. 5. Pixel ray projection into the feature plane to recover 3-D information.

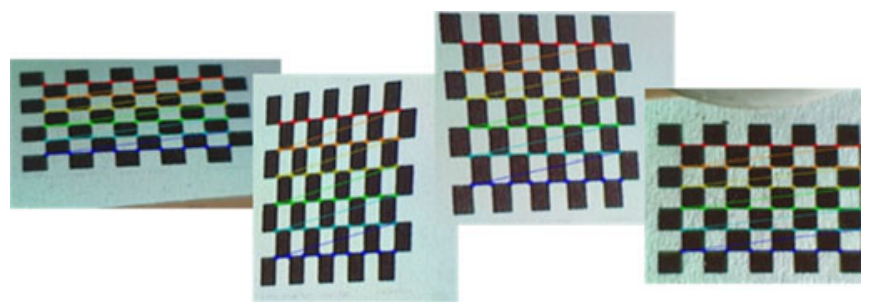

Fig. 6. Different pictures from different camera positions of the calibration pattern.

the bead width, the plane that contains the two edges of the bead is a plain in which the welding is taking place.

Fig. 5 shows this concept in details. To project the pixel into the feature plane and recover the 3-D coordinate, the intersection between the pixel ray line and the plane must be found. The equation of the pixel projection line can be solved using the intrinsic and extrinsic parameters obtained previously with the camera calibration.

The vector equation of a line can be expressed as

$$
\vec{r}=\overrightarrow{r_{0}}+\lambda \cdot \vec{m}
$$

where $\overrightarrow{r_{0}}$ is a point on the line, and $\vec{m}$ is the line direction. By this means $\vec{r}$ would contain any point on the line by varying the distance $\lambda \in(-\infty,+\infty)$ from $\overrightarrow{r_{0}}$ to this point. To find the vector equation of the pixel ray line in nozzle coordinates, $\overrightarrow{r_{0_{N}}}$ can be calculated as the camera position, found in the previous section, expressed as

$$
\overrightarrow{r_{0_{N}}}=t_{\mathrm{NC}}=-R_{\mathrm{NC}} \cdot t_{\mathrm{CN}} .
$$

To calculate the direction vector $\vec{m}$, the point $\overrightarrow{r_{1}}$ shown in Fig. 6 can be used. Note that this point in the camera coordinate system corresponds to

$$
\overrightarrow{r_{1_{C}}}=\left[\begin{array}{l}
u \\
v \\
f
\end{array}\right] .
$$

To transform $\overrightarrow{r_{1_{C}}}$ from the camera coordinate system to the nozzle coordinate system, the rotation matrix and translation vector are used. The transform can be written as

$$
\overrightarrow{r_{1_{N}}}=R_{\mathrm{NC}}\left[\begin{array}{l}
u \\
v \\
f
\end{array}\right]+t_{\mathrm{NC}} \text {. }
$$


Hence, the direction vector can be calculated by $\vec{m}=\overrightarrow{r_{1_{N}}}-\overrightarrow{r_{0_{N}}}=\left(R_{\mathrm{NC}}\left[\begin{array}{c}u \\ v \\ f\end{array}\right]+t_{\mathrm{NC}}\right)-t_{\mathrm{NC}}=R_{\mathrm{NC}}\left[\begin{array}{l}u \\ v \\ f\end{array}\right]$.

Next, once the pixel ray line equation, that defines all the possible 3-D positions of the 2-D pixel, is determined, the intersection with the feature plane is used to recover the exact location among all the infinite possibilities. Having a general normalized plane

$$
\vec{n} \cdot \vec{r}=d
$$

where $\vec{n}$ is a unit vector normal to the plane and $d$ is the minimum distance from the plane to the origin. Substituting (16) into (21), the line-plane intersection can be found; the value of $\lambda$ can be calculated by

$$
\lambda=\frac{d-\overrightarrow{r_{0_{N}}} \cdot \vec{n}}{\vec{m} \cdot \vec{n}} .
$$

\section{Results And Discussions}

\section{A. Intrinsic and Distortion Parameters}

This section corresponds to steps 4 and 5 of the camera calibration methodology explained in Section III-B. A total of 12 positions of the camera have been tested to estimate the intrinsic and distortion coefficients. The resolution of the image is $800 \times 600$ pixels. Fig. 6 shows four examples that illustrate the detect feature points on the calibration checkerboard to perform the calibration. Note that the world origin would correspond to the first red point from the top left. Then, the axis will be defined depending on how the successive points are defined in the 3-D space. Because the calibration parameters were calculated by averaging the results from different calibration images, more calibration images usually produce more accurate results. All calibration steps and image processing algorithms were implemented through the Visual Studio $\mathrm{C}++2015$ and OpenCV library.

Assuming square pixels, the detected intrinsic parameters (unit: pixel) are

$$
K=\left[\begin{array}{ccc}
8077.7 & 0 & 399.5 \\
0 & 8077.7 & 299.5 \\
0 & 0 & 1
\end{array}\right]
$$

Based on this matrix, it can be deduced that $f_{x}=f_{y}=$ 8077.7 pixel and that the center of the image with respect to the upper left corner is $C_{x}=399.5$ pixel and $C_{y}=299.5$ pixel.

The detected camera distortion parameters are

$$
\begin{aligned}
& k_{1}=2.95 \\
& k_{2}=1755.86 \\
& k_{3}=5.31 .
\end{aligned}
$$

The raw images can then be applied by (3) and (4) based on the identified parameters shown in (24) to reduce the distortion, by which means the reliability of the measurements produced by the next steps can be improved. The right picture of Fig. 7 shows
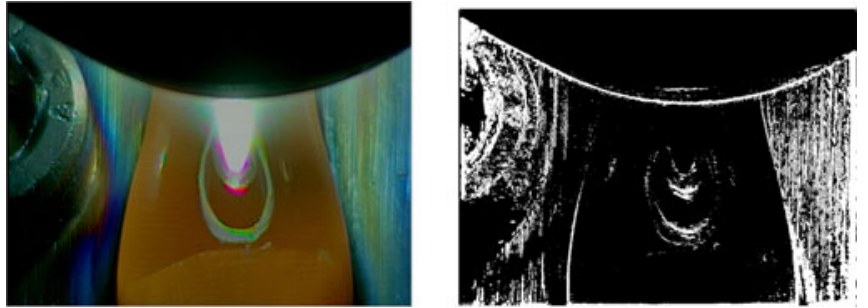

Fig. 7. (Left): A raw image. (Right): The difference between the raw and the undistorted image.

a difference image between a raw image and the corresponding undistorted image in a binary form. A clear shift can be noticed on the boundary of the weld pool, which will affect the following measurement.

\section{B. Extrinsic Parameters}

Once the intrinsic parameters and distortion parameters are calculated, the position of the camera was fixed on the working place and another picture of the grid was taken to calculate the extrinsic parameters, as stated in steps 6 and 7 in Section III-B.

The position of the nozzle of the $\mathrm{CNC}$ machine has been used to find the orange transformation in Fig. 4. Fig. 7 shows a picture captured from the top position where the coordinates of the nozzle (unit: $\mathrm{mm}$ ) are

$$
t_{\mathrm{CNC} 1}=\left\{\begin{array}{lll}
198.49 & 266.65 & 244.44
\end{array}\right\} .
$$

Knowing that the position of the CNC in the world coordinate (unit: $\mathrm{mm}$ ) can be converted to

$$
t_{\mathrm{CNC} 2}=\left\{\begin{array}{lll}
180.29 & 257.68 & 247.33
\end{array}\right\}
$$

the translation vector from the world coordinate system to the nozzle coordinate system (the orange transformation) (unit: $\mathrm{mm}$ ) can be calculated by

$$
t_{\mathrm{WN}}=t_{\mathrm{CNC} 1}-t_{\mathrm{CNC} 2}=\left\{\begin{array}{lll}
18.20 & 8.97 & -2.89
\end{array}\right\} .
$$

The extrinsic parameters, composed by the rotation and translation from the camera coordinate to the world coordinate (the blue transformation in Fig. 4), are shown in (28) and (29), respectively

$$
\begin{aligned}
& R_{\mathrm{CW}}=\left[\begin{array}{ccc}
0.9989 & 0.0135 & -0.0446 \\
0.0018 & 0.9450 & 0.3270 \\
0.0466 & -0.3268 & 0.9440
\end{array}\right] \\
& t_{\mathrm{CW}}=\{-6.46 \mathrm{~mm} \quad-2.73 \mathrm{~mm} \quad 219.71 \mathrm{~mm}\} \text {. }
\end{aligned}
$$

Since the nozzle does not rotate with respect to the world coordinate system, finally, the camera rotation and translation, with respect to the nozzle coordinate system, can be found in (30) and (31), respectively

$$
\begin{aligned}
R_{\mathrm{NC}} & =\left[\begin{array}{ccc}
0.9989 & 0.0018 & 0.0466 \\
0.0135 & 0.9450 & 0.3268 \\
-0.0446 & -0.3270 & 0.9440
\end{array}\right] \\
t_{\mathrm{NC}} & =\left\{\begin{array}{lll}
-21.97 \mathrm{~mm} & -65.49 \mathrm{~mm}-203.90 \mathrm{~mm}\} .
\end{array}\right.
\end{aligned}
$$




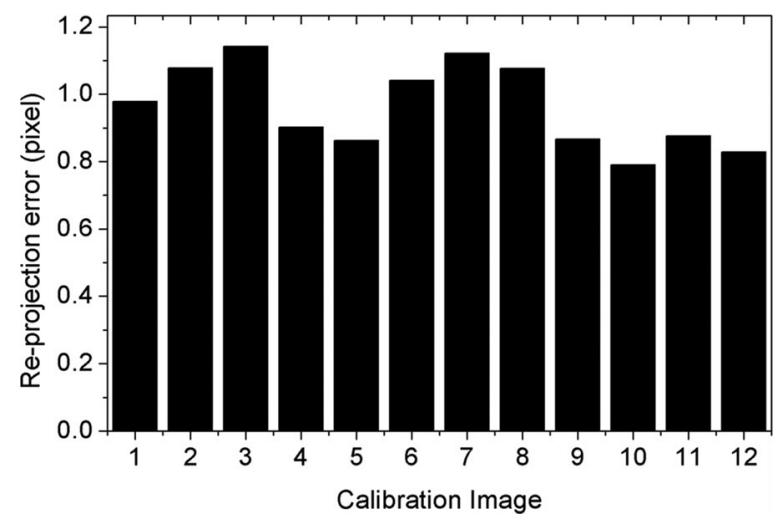

Fig. 8. Reprojection error for each image used in calibration.

The rotation matrix in (30) can also be written as Euler angles, as shown in (32) and (33)

$$
\begin{aligned}
\psi_{1} & =19.11^{\circ} \\
\theta_{1} & =2.55^{\circ} \\
\phi_{1} & =0.78^{\circ} \\
\psi_{2} & =-160.89^{\circ} \\
\theta_{2} & =177.44^{\circ} \\
\phi_{2} & =-179.22^{\circ} .
\end{aligned}
$$

The reprojection errors of the calibration process are used to evaluate the accuracy of the calibration. It consists of projecting the 3-D calibration grid points using the estimated calibration parameters, and then measuring the mean square error between the projected points and the original points in the image. Totally 12 tests with different camera positions have been conducted to estimate the extrinsic parameters. Fig. 8 shows the plot of the reprojection error for every calibration image. Variation of error has been observed but in a relatively small range. The averaged error is $0.964 \pm 0.124$ pixel. Note the camera positions for the 12 cases are different, which indicates that the extrinsic parameters are different and the scales between the world coordinate and the image coordinate are different. The error in $\mathrm{mm}$ would only be valid for a particular case; consequently, it is believed that providing the reprojection error in pixels is more general because it is independent on the scale/distance of the object that is being recorded.

\section{Width Tracking}

The proposed imaging and calibration system aim to accommodate measurement of different properties of weld pool. This section presents a case study to measure the width of bead to demonstrate this system.

The following two assumptions are made to obtain good results using this procedure.

1) The edge of the bead can be found in images.

2) The desired boundary movement from one frame to another is smooth. This assumption is valid for most cases because the change in the bead width will hardly be abrupt.
The steps to measure the bead width can be described as follows.

1) Apply the distortion parameters shown in (17) to correct the distortion.

2) Initialize edge points and apply a window: To know where to start tracking the edges of the bead, two edge points are initialized. These points are initialized manually by clicking on the image. The first click determines the $x$ and $y$ coordinates of the first point as well as the $y$-coordinate of the second point. The second click determines the $x$ coordinate of the second point. Based on these points, two windows are applied, one for each of the edges, to reduce the region of interest allowing to reduce the amount of calculations to process the image and find the edges.

3) Image enhancement: The mean shift filter is applied to reduce noise, increase the chance to find the edge, and improve the reliability of measurement. The mean shift filter is an iterative filter, which basically produces a color segmentation of the image by processing a certain amount of neighbor pixels with a certain range in the color acceptance to make one particular color region to grow progressively.

4) Edge detection: The Canny edge detector is used to find reliable edges in an image.

5) Thresholding: Otsu's thresholding method is employed in this paper to remove weak edges and preserve the strong ones.

6) Line extraction: The Hough Transform is employed to find lines in a binary image.

7) Line selection: After obtaining a range of possible lines from the Hough Transform, a decision has to be made to select which of the extracted lines correspond to the boundary of the bead. This step is essential because the presence of noise in the image introduces false edges, which must be filtered before the landmark detection. To address this, several assumptions are made, which are as follows:

a) only the most reliable ones given by the Hough Transform will be analyzed;

b) the most reliable line will always be the one with the middle point closer to the previous bead corner if this distance is inside a certain threshold; and

c) if the lines obtained are outside the threshold, the most reliable line will tend to be the one inside the weld pool, that is at the right side for the left edge, and at the left side for the right edge.

8) Landmark detection: The middle point on the selected lines will be used as the landmarks to calculate the width.

9) Calculate the width in the unit of "mm" by applying the extrinsic parameters shown in (23) and (24) identified from the calibration procedure.

Fig. 9 illustrates the routine to measure the bead width and the intermediate images corresponding to each step.

Fig. 10 plots the results of bead width measurement for 40 consecutive frames from two tests using the proposed calibration system and image analysis method, plotted by the solid curves. Fig. 11 shows a snapshot of the bead width 


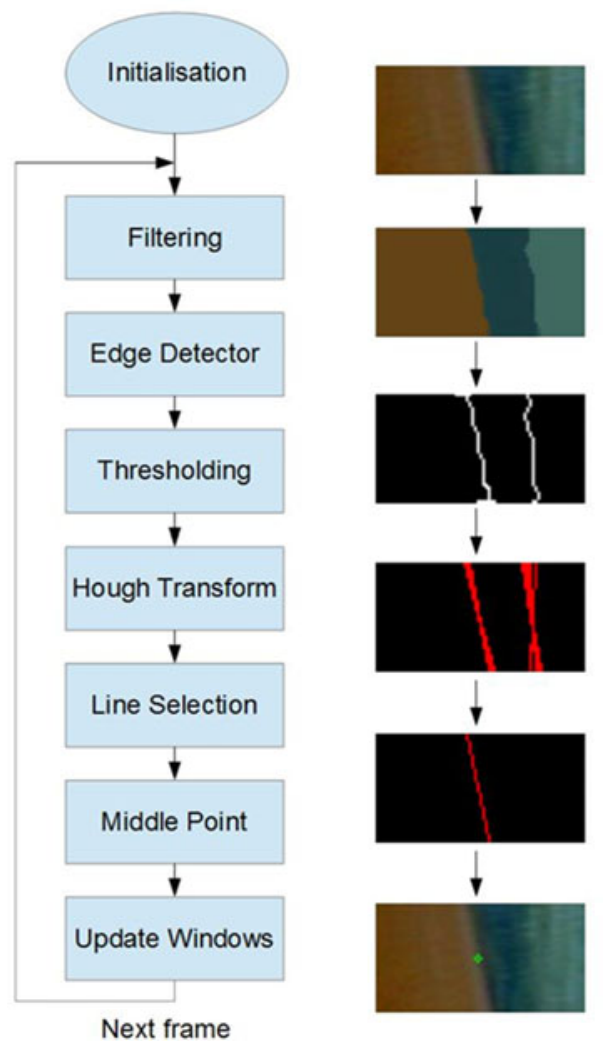

Fig. 9. Illustration of the developed width tracking method.

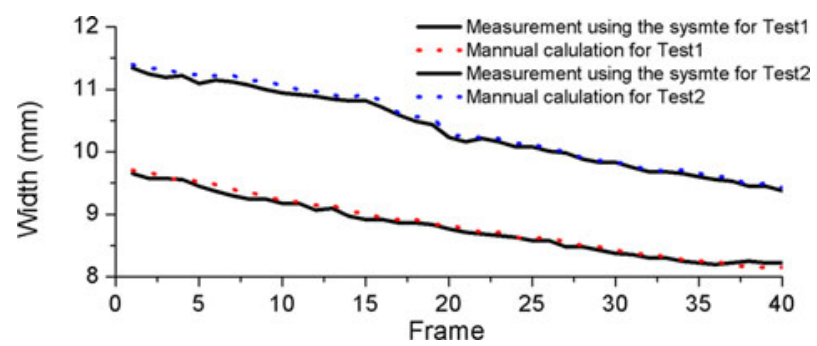

Fig. 10. Width measurement for 40 consecutive frames from two tests.

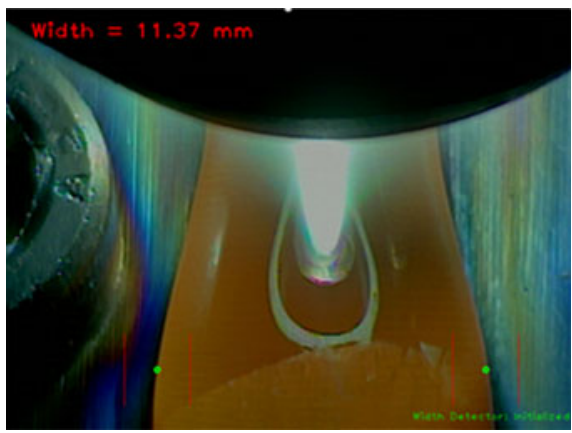

Fig. 11. Width measurements for test 1 at frame 1.

measurement. To evaluate the accuracy of measurement, the bead width was manually measured based on the sampled images and the results are plotted by the dotted curves in Fig. 10. Visual inspection indicates a very good approximation of the ground truth. The average errors for Tests 1 and 2 are $0.052 \pm 0.030 \mathrm{~mm}$ and $0.062 \pm 0.034 \mathrm{~mm}$, respectively, or $1.48 \pm 1.82$ and $2.40 \pm 1.35$ pixels, respectively.

Although this case study focuses on one parameter only, the proposed imaging system can measure multiple parameters at the same time. For example, if the set up shown in Fig. 2(b) is used, both the width of bead and the position of wire (e.g., angle of the wire) can be measured. This case study only demonstrates how to use the proposed system to measure a parameter. Such a methodology can be easily extended to measure other parameters, while the difference would be the feature detection using image processing methods.

\section{CONCLUSION}

The main goal of this research was to investigate how to sense a PAW process through a low-cost computer vision technique in an easy-to-use approach. To achieve this goal, this paper proposed a passive imaging system, which includes data acquisition setup, a novel calibration routine, feature extraction, and measurement. This system allows the user to track the weld pool geometry online during the welding processing, which can be feedbacked to the controller of experimental parameters to better control this process. The verification tests showed that the averaged error of calibration is less than 1 pixel. A case study to track the bead width was presented in this paper to demonstrate how the proposed system measures the geometry of the weld pool. A novel routine of feature extraction using image processing technique was proposed. The results showed that the proposed system can effectively track the dynamics of the bead width.

The main novelty of the research was the calibration methodology, specifically for PAW, which allows transforming measurements from pixel to the real-word unit automatically through an easy-to-use routine and accurately through compensating camera distortion. The proposed calibration moves the camera with respect to the calibration pattern on the deposition position instead of moving the calibration pattern due to the space limitation in the CNC machine. Because the position of the camera with respect to the nozzle was always the same, the position of nozzle and the estimated parameters were then used to localize the camera position. The standard calibration process was tailored to better fit the monitoring of the PAW process through such unique changes.

The proposed system features with the following key advantages.

1) The camera distortion was considered to improve the measurement accuracy.

2) Having the whole camera model allows the fast and accurate calculation of the camera position in 3-D. This can be especially useful to set up the experimental condition and to perform further experiments by changing the camera positions in order to improve the image quality or to measure other features from the process.

3) No laser illumination was required, and the whole imaging system was relatively less experience.

The proposed calibration process assumes that the camera has square pixels $\left(f_{x}=f_{y}\right)$, which fits to most of the cameras. If the pixels were not exactly square, the process to estimate intrinsic 
parameters will not be affected. Two independent parameters $f_{x}$ and $f_{y}$ will be estimated. However, the current equations (18)-(20) will be affected and need to be extended, which will be one of the future works.

This system is ready to accommodate measurement of different features either by changing the camera or camera position. Futures works will focus on the following:

1) identifying the optimal camera position to measure multiple important parameters of weld pool;

2) 3-D surface reconstruction of the whole weld pool; and

3) the influence of different control parameters to the geometry of weld pool.

\section{REFERENCES}

[1] S. W. Williams, F. Martina, A. C. Addison, J. Ding, G. Pardal, and P. Colegrove, "Wire + arc additive manufacturing," Mater. Sci. Technol., vol. 32, no. 7, pp. 641-647, 2016.

[2] R. Kovacevic, Y. Zhang, and L. Li, "Monitoring of weld joint penetration based on weld pool geometrical appearance," Weld. J., vol. 38, pp. 317$329,1996$.

[3] L. O. Vilarinho, B. Lucas, and M. Houghton, "Dedicated near-infrared vision system for monitoring welding processes," in Proc. 20th Int. Congr. Mech. Eng., Gramado, Brazil, 2009, pp. 7-13.

[4] H. Song and Y. Zhang, "Measurement and analysis of three-dimensional specular gas tungsten arc weld pool surface," Weld. J., vol. 87, pp. 85-95, 2008

[5] Z. Wang, "An imaging and measurement system for robust reconstruction of weld pool during arc welding," IEEE Trans. Ind. Electron., vol. 62, no. 8, pp. 5109-5118, Aug. 2015.

[6] J. Zeshi, L. Haichao, J. Guoquing, and G. Hongming, "Dynamic non-linear modeling of 3D weld pool surface in GTAW," Robot. Comput.-Integr. Manuf., vol. 39, pp. 1-8, 2016.

[7] W. Lucas, D. Bertasco, G. Melton, J. Smith, and C. Balfour, "Real-time vision-based control of weld pool size," Weld. Int., vol. 26, no. 4, pp. $243-$ $250,2012$.

[8] C. Wu, J. Gao, X. Liu, and Y. Zhao, "Vision-based measurement of weld pool constant-current gas tungsten arc welding," Proc. Inst. Mech. Eng., J. Eng. Manuf., vol. 217, pp. 879-882, 2003.

[9] J. Liu, Z. Fan, S. I. Olsen, K. H. Christensen, and J. K. Kristensen, “A real-time passive vision system for robotic arc welding," in Proc. IEEE Int. Conf. Autom. Sci. Eng., 2015, pp. 389-394.

[10] J. Xiong, G. Zhang, Z. Qiu, and Y. Li, "Vision sensing and bead width control of single-bead multi-layer part: Material and energy saving in GMAW-based rapid manufacturing," J. Cleaner Prod., vol. 41, pp. 82-88, 2012

[11] J. Xiong and G. Zhang, "Adaptive control of deposited height in GMAWbased layer additive manufacturing," J. Mater. Process. Technol., vol. 214, pp. 962-968, 2013.

[12] J. Xiong and G. Zhang, "Online measurement of bead geometry in GMAW-based additive manufacturing using passive vision," Meas. Sci. Technol., vol. 24, no. 11, 2013, Art. no. 115103.

[13] Q. Zhan, Y. Liang, J. Ding, and S. Williams, "A wire deflection detection method based on image processing in wire + arc additive manufacturing," Int. J. Adv. Manuf. Technol., vol. 89, pp. 755-763, 2017.

[14] P. Ghanty et al., "Artificial neural network approach for estimating weld bead width and depth of penetration from infrared thermal image of weld pool," Sci. Technol. Weld. Joining, vol. 13, no. 4, pp. 395-401, 2016.

[15] Z. Chen and X. Gao, "Detection of weld pool width using infrared imaging during high-power fiber laser welding of type 304 austenitic stainless steel," Int. J. Adv. Manuf. Technol., vol. 74, no. 9, pp. 1247-1254, 2014.

[16] F. Bonnacorso, L. Cantelli, and G. Muscato, "An arc welding robot control for a shaped metal deposition plant: Modular software interface and sensors," IEEE Trans. Ind. Electron., vol. 58, no. 8, pp. 3126-3132, Aug. 2011.

[17] J. Xiong, Z. Yin, and W. Zhang, "Closed-loop control of variable layer width for thin-walled parts in wire and arc additive manufacturing," $J$. Mater. Process. Technol., vol. 233, pp. 100-106, 2016.

[18] F. Martina, J. Mehnen, S. Williams, P. Colegrove, and F. Wang, "Investigation of the benefits of plasma deposition for the additive layer manufacture of Ti-6Al-4V," J. Mater. Process. Technol., vol. 212, pp. 1377-1386, 2012.
[19] G. G. Slabaugh, "Computing Euler angles from rotation matrix," accessed on Nov. 15, 2016. [Online]. Available: http://www.gregslabaugh. name/publications/euler.pdf

[20] C. X. Zhao, I. M. Richardson, S. Kenjeres, C. R. Kleijn, and Z. Saldi, "A stereo vision method for tracking particle flow on the weld pool surface," J. Appl. Phys., vol. 105, 2009, Art. no. 123104.

[21] X. Z. Chen, Y. M. Huang, and S. B. Chen, "Model analysis and experimental technique on computing accuracy of seam spatial position information based on stereo vision for welding robot," Ind. Robot, Int. J., vol. 39, no. 4, pp. 349-356, 2012.

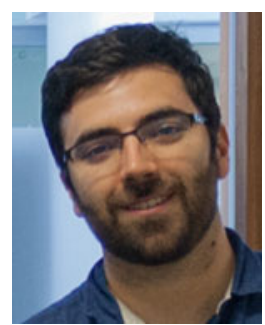

Tomas Font comas was born in Spain. He received the M.Sc. degree in computational and software techniques in engineering from Cranfield University, Cranfield, U.K., in 2016.

He currently runs a company called Videoassist/Equipo in Spain. His current research focuses on digital signal and image processing with applications in conditional monitoring.

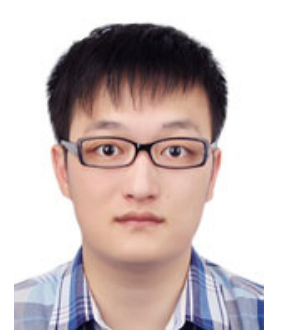

Chenglei Diao was born in Jiangsu, China. He received the M.Eng. degree in mechanical engineering from the University of Cambridge, Cambridge, U.K., in 2016. He is currently working toward the Ph.D. degree in welding engineering at the Welding Engineering and Laser Processing Centre, Cranfield University, Cranfield, U.K.

During his undergraduate studies, he served internships with COMAC, FFT Produktionssysteme $\mathrm{GmbH} \&$ Co. KG, and Aritex.

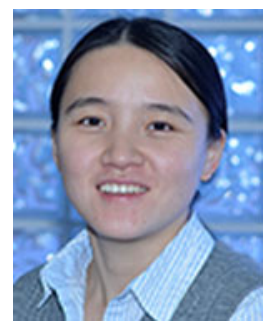

Jialuo Ding was born in Qinhuangdao, China. She received the Ph.D. degree in welding engineering from Cranfield University, Cranfield, U.K., in 2012.

After receiving the Ph.D. degree, she became a Research Fellow in the Welding Engineering and Laser Processing Centre, Cranfield University, and continued her research on the wire + arc additive manufacturing (WAAM) process from 2012 to 2015 . In 2015, she was promoted to Senior Research Fellow in additive manufacturing. Her research interests include thermal-mechanical modeling of the WAAM, WAAM software development, process control and automation of the WAAM process, and manufacturing strategy for building largescale components with WAAM.

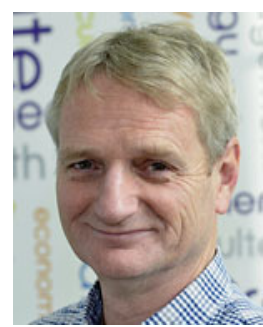

Stewart Williams was born in Lichfield, U.K. He received the $\mathrm{Ph} . \mathrm{D}$. degree in laser physics from the University of London, London, U.K., in 1981.

$\mathrm{He}$ is currently the Director of the Welding Engineering and Laser Processing Centre, Cranfield University, Cranfield, U.K., with research focused on large-scale additive manufacture and laser processing. He was previously a Development Engineer with Edinburgh Instruments and a Researcher and Group Leader with BAE Systems.

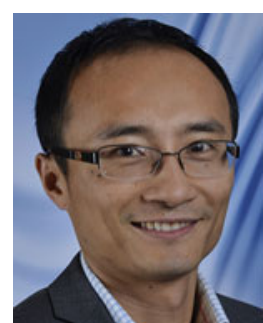

Yifan Zhao was born in Zhejiang, China. He received the Ph.D. degree in automatic control and system engineering from the University of Sheffield, Sheffield, U.K., in 2007.

$\mathrm{He}$ is currently a Lecturer in image and signal processing and degradation assessment at Cranfield University, Cranfield, U.K. His research interests include computer-vision-based process monitoring, super resolution, active thermography, and nonlinear system identification. 
2017-03-22

\section{A passive imaging system for geometry measurement for the plasma arc welding process}

Font comas, Tomas

Institute of Electrical and Electronics Engineers

Tomas Font comas, Chenglei Diao, Jialuo Ding, Stewart Williams and Yifan Zhao. A passive imaging system for geometry measurement for the plasma arc welding process. IEEE

Transactions on Industrial Electronics, Vol. 64, Issue 9, pp. 7201-7209

http://dx.doi.org/10.1109/TIE.2017.2686349

Downloaded from Cranfield Library Services E-Repository 